\title{
On A Logically Consistent Cosmological Model Based on Buddhist Philosophy
}

\author{
Wei-Cheng Cui ${ }^{1,2 *}$ \\ ${ }^{1}$ School of Engineering, Westlake University, China \\ ${ }^{2}$ Shanghai Engineering Research Center of Hadal Science and Technology, Shanghai Ocean University, China
}

Submission: February 27, 2019; Published: March 06, 2019

"Corresponding author: Weicheng Cui, Chair professor at Westlake University, China and adjunct professor at Shanghai Ocean University, China

\section{Abstract}

The Big Bang theory is the currently most prevailing cosmological model for the observable universe, but it still exists many un-answered questions such as the existence of the Singularity and the reason for a sudden explosion. Many people are still working on the improvement of the Big-Bang model or the construction of other totally new cosmological models. In this paper, a novel cosmological model based on Buddhist philosophy is proposed and from this author's judgement, it seems conceptually clear and logically consistent and it can explain many phenomena which belongs to the frontier problems of modern sciences.

Keywords: Universe; Cosmology; The Big-Bang Cosmological Model (BBCM); The Buddhist Cosmological Model (BCM)

\section{Introduction}

Where did we come from? Where should we go after dying? Who am I? When did the universe begin? How big is it? Will it end? Does the universe operate according to some laws or randomly? These and many other similar questions have been explored since very ancient times by many people including scientists, philosophers, religionists, and many theories have been proposed [1]. Today, the consensus among scientists, astronomers and cosmologists is the Big Bang Theory also known as the Big-Bang Cosmological Model (BBCM). The basics of the Big Bang theory are simple. In short, the Big Bang hypothesis states that all the current and past matter in the Universe came into existence at the same time, roughly 15 billion years ago. At this time, all matter was compacted into a very small ball with infinite density and intense heat called a Singularity. Suddenly, the Singularity began expanding, and the universe as we know it began.

This model has been confronted to a variety of observations that allow one to reconstruct its expansion history, its thermal history and the structuration of matter. Hence, what we refer to as the big-bang cosmological model (BBCM) today is radically different from what one may have had in mind a century ago. And I believe that a hundred years later, this big-bang model might also be radically different from what it is now. Burago pointed out that at least two obvious questions exist in the Big-Bang model [2].

The first question remained as to how the matter and energy were in this superdense elementary particle? It is considered incorrect to ask, what was around this particle before the explosion and whether the universe does expand? Because space and time in the universe also arose as a result of the Big Bang. It is assumed that protons, neutrons, positrons, electrons and other long-lived elementary particles formed 15 billion years ago and have reached our days unchanged. The second question arose from the insolvency of ideas about the explosion of a kind of "cosmic egg", which was the explosion of the largest nuclear bomb. This point of view boils down to the assertion that "space" exploded, and not a material object. At the same time, the authors of this idea do not bother explaining what they think is a "space" and what can explode in an empty space? Therefore, many people are still working on the improvement of the Big-Bang model or the construction of other totally new cosmological models.

All the modern sciences including the BBCM are based on the materialism which holds that matter is the fundamental substance in nature, and that all things, including mental aspects and consciousness, are by-products or epiphenomena of material processes (the biochemistry of the human brain and nervous system, for example) without which they cannot exist. According to this doctrine the material creates and determines consciousness, not vice versa. Now this philosophy cannot explain many life phenomena, e.g. [3]. Sheldrake [4] has discussed in quite detail about the ten dogmas of modern science based on materialism and some people even claim that it is the time now 
to end the materialism, e.g. [5]. The most difficult question for the materialism believers is how the first matter comes from and what is the source of forces which cause every object to move in the universe.

In this paper a novel cosmological model based on Buddhist philosophy is proposed and therefore, we call this model as the Buddhist Cosmological Model (BCM). From this author's judgement, it seems conceptually clear and logically consistent and it can explain many phenomena which belongs to the frontier problems of modern sciences. It is the author's wish that critical comments can be received by publishing this paper.

\section{The Logically Consistent Cosmological Model}

It must be emphasized that all the theories we are talking about even including the concepts of space and time are created by our human beings and they are only of meaning to our human beings and not animals. I am sure quite a lot of types of animals also have verbal languages which we do not understand. Now we are accustomed to describing any phenomenon in the space-time framework. In this paper, I do not provide any new definitions for space and time. A quote of Kant is: "Space and time are the framework within which the mind is constrained to construct its experience of reality" [6].

In the BBCM, it is very clear that the universe is finite in time but unclear whether it is finite or infinite in space. In the present $\mathrm{BCM}$, it is specifically defined that the universe is of infinite nature in both space and time. The world is defined of finite nature in both space and time and their relationship is expressed by the following equation:

$$
\text { Universe }=\sum_{i=1}^{\infty} \text { world }_{i}
$$

Each world is cyclically operated according to the process of formation, the steady state, deterioration and explosion to emptiness. In BCM, the Big Bang is the origin of the world but not the universe. Using this definition, the awkward question of the origin of the universe can be avoided.

Different from materialism which assumed matter is the fundamental substance in nature, the Buddhism assumed that the energy is the fundamental essence of the universe. The universe consists of two types of energy, explicit energy and dark energy. Dark energy is defined as the essence of a life corresponding to the alaya consciousness in Buddhist theory [7,8], it has the capability to accumulate the explicit energy into matter and decompose the matter into explicit energy. Explicit energy is the lifeless energy which can be accumulated by the alaya consciousness to be explicit matter or dark matter and then to bodies or objects. The level can be sensed by our human beings is defined as the explicit matter and the rest is defined as the dark matter.

\section{Both Explicit Energy and Dark Energy in the Universe Always Exists and Satisfies the Conservation Law, but not the Matter}

This is the fundamental assumption made in BCM. Through this assumption, the awkward question of the origin of life in the universe can also be avoided. All matter is accumulated by the dark energy (the alaya consciousness). This answers how the first matter comes from and the source of all types of forces in the universe which cause all the movements and changes of the universe. However, it must be emphasized here that what we have observed by our eyes are only small part in the world we are living and not the whole universe. It is the over-claim that this is the whole universe, and this is similar as a frog sitting at the bottom of a well.

There are three types of life in the universe, the alaya consciousness only, the alaya consciousness with an insensible body (also dark matter to our human being) and the alaya consciousness with a sensible body (being animals and human beings) and these three types of lives can also be divided into six categories according to their happiness, Heaven, Asura, Human being, Animal, Ghost, Hell. Only human beings and animals are with a sensible body by our human beings. Non-existence of other types of lives is also a belief or an over-claim rather than a scientific proof. Lives with only the alaya consciousness only exists in the Heaven, they are the highest level of life in the universe while the lives in the Hell is the lowest level in the universe. So according to this BCM, human beings are not the most intelligent creatures in the universe but in the middle level. All the matter used by other four types of lives and together with their bodies belong to dark matter.

\section{Everything in the Universe Including all the Worlds and Each Individual Life is Operated According to the Causal-Effect Law}

This can be regarded as the second assumption of this BCM. For any lifeless object in the world we are living, no matter whether we can see (explicit matter) or not (dark matter), it will experience the cycle of formation, the steady state, deterioration and destroy and for each individual life, no matter whether we can see (human beings and animals) or not (other four types of lives), she/he will be reincarnated within the six types of lives in the universe. Here it must be pointed out that according to the causal-effect law, reincarnation of my live is not confined to this world I am living, but in the whole universe. Even for the alaya consciousness only life in the Heaven, she/he will also have a life span and can be reincarnated into other five types with a body in the universe, not necessarily in the same world as he/she lived in a previous life. Reincarnation of all lives is a law of nature [9]. So, in this BCM, parents provide only the bodies to their children and not the life. We are very soon reached a stage that we can clone our bodies, but the essence of life does not change, and any lives produced this way should have the same rights as us.

Human's consciousness includes 8 types, they are consciousness at eyes, ears, nose, tongue, body and mind, the manas consciousness, and finally the alaya consciousness. When 
one is dying, the consciousness at eyes, ears, nose, tongue, body and mind will be lost. The function of the seventh consciousness whose formal name is called the manas consciousness, is the bridge between the former six consciousness with the eigth whose formal name is called the alaya consciousness. Only the alaya consciousness will continue to exist and it is the source of life forces and it stores all the karmas in the previous life history. The dying process is a process of the separation of the alaya consciousness with the body. For the detailed theory of Buddhism, one can refer to references $[7,8]$

\section{Explanation Power of BCM}

Through my education and scientific training, I cannot see any logical problem with BCM, it gives a clear definition to all the concepts used and state clearly only two fundamental assumptions are needed. One is the existence of energy, different from the materialist world view, there are basically two types of energy, one is the explicit energy which is the essence of all matter, no matter explicit matter which we can see or feel or the dark matter which we cannot see or feel, and the other is dark energy which is the essence of life. This is called the alaya consciousness in the Buddhist philosophy. The other assumption is that everything in the universe follows the causal-effect law. How to reveal this law for each object is the mission of our scientific researches. With this model, it avoids many awkward questions related to the origin of universe and life in the universe and it answers the important questions of the origin of matter and the source of forces behind. It has automatically revealed the most difficult part of modern physics about dark matter and dark energy. It can easily explain many other phenomena in the world we have observed. To my own knowledge, this model seems to be able to explain all the anomalous phenomena mentioned in Ref. [3]. In this section, further explanation powers to some important problems are shown. Of course, it is impossible for one paper to cover every aspect and these can just be regarded as a demonstration.

\section{Origin of the Earth and Life on the Earth}

In this $\mathrm{BCM}$, it is natural that any star like the earth was created by many lives in the universe and when the condition suits, the relevant alaya consciousness will enter a body and that is the origin of life on the earth and any stars.

\section{Existence of the Extraterrestrial Life}

It is also natural that the extraterrestrial life exists everywhere in the universe, however, up to now, we have not found the macro creatures which we can see in our neibouring stars yet. But it cannot refuse the possibility that other types of lives exist which we cannot see now since a very high percent of dark matter we do not know. In this BCM, the dark matter is those lives and their material stuff for living. As a matter of the fact, even on the earth, other four types of lives may also exist although we could not see them by conventional means but there are some other means which can communicate with them. One approach is through a mediumship described in Chapter 10 of Ref. [3]. But this approach may only communicate with lives in the Ghost, whether a medium is able to communicate with lives in the Heaven and Hell is a problem for further study.

\section{Criterion of a Perfect Human Life}

For believers in materialism, life only has one term. It is hard to define a perfect state. Now, most of the people assess some's success by how much money he owned. In order to earn more money, he works hard and fights with others due to the resource limit. One day when he is dying, he cannot take any money away. He should not know anything whether his money has given to his children or others. Therefore, it is hard to see the difference between a hard-working person and a lazy person. The fighting for limited resources between human beings cause a lot of social problems. In BCM, it is clear to make the next life in a higher level is much more important than earning money in this life. The highest life is the Budda which every person can be if he always does good things $[7,8]$. This could make the society more harmony than the current materialism belief.

\section{How to Know the Essence of Universe}

In $\mathrm{BCM}$, there are two methods for human beings knowing the operational laws of the world, one is the current knowledge learning method. This is based on the principle of a blind man feeling an elephant. We are approaching the truth, but it hardly knows the truth. The second method is to open your "eyes" to see the elephant. However, how to open this "eye" which can see the truth needs to be practiced. In Buddhist religion, there are many methods to develop this capability for different individuals. In general, meditation is one of the main methods to be used [10].

\section{Artificial Intelligence and Human Consciousness}

Nowadays, artificial intelligence progresses very fast and it has defeated human beings in the game of Go [11]. Some people started to worry that one day artificial intelligence will be more powerful than human beings. According to BCM, human beings can only make bodies but not the alaya consciousness. The unique property of the alaya consciousness is its ability of "self-knowing oneself", that is, in the moment of perceiving the outside world, there is a kind of vivid and clear, self-awareness. This kind of intrinsic character of the present, clear and self-awareness, in the Dharma, is called the "self-certification of the alaya consciousness" or the meaning of "self-knowing oneself". Such a kind of conscious and conscious experience is the fundamental difference between our human beings and robots.

\section{Theory of everything}

Whether the world works regularly or not, and whether the nature of the world is knowable or unknowable, Einstein and Bohr have debated these two questions for more than 40 years, no one was persuaded by the other until their death. After that, physicists divided into two camps to continue the debate. The detailed story can be found in many articles and books, e.g. [12,13]. Einstein believes that the world works regularly, and the operational 
law can be revealed by our scientific research. So, he wished to establish a theory of everything (TOE) but he failed. Many others are continuing this direction.

In a previous paper by this author, a simple idea on the unification of Einstein-Bohr Controversy was presented [14]. My main point is that the controversy was caused by Bohr's overclaim and there is no contradiction between them. Philosophically, we should believe that (1) the universe including macro and micro worlds always works regularly or with rules, and (2) these rules can always be revealed by us humans. Practically, due to the complexity of the actual problem and the time limit of our human life, we may not be able to reveal the real rules of the system operation in our lifetime, especially it is hard for us to identify all the influencing factors. If all the influencing factors have not been identified for a system, then the transfer function is an approximate format. We can define those ignored factors as hidden variables. Because of the existence of hidden variables and the approximate nature of the transfer function, there is no one to one correspondence between input vector and output value, and the system is uncertain.

In this case, we should apply a theory who is able to handle uncertainty such as probability and statistics. In conclusion, for systems with no hidden variables, the transfer function can be given precisely using deterministic mathematics. For systems with hidden variables, the mathematics with uncertainty, such as the theory of probability and statistics, should be applied. There is no inconsistency to be debated. The deepening research can be carried out in two directions. One is to study the mechanism more precisely and reduce or eliminate hidden variables. The second is to study its application. On the premise of only considering the currently accepted explicit influencing factors, probability and statistics theory is applied to improve the reliability of prediction as much as possible under the condition of existing information or collecting more information only on these factors.

Philosophically speaking, there is no need to make the claims that (1) there is no rule in the micro world operation and (2) there exists irreducible uncertainty or objective uncertainty. From this author's knowledge, Shen Zhiyuan [15] has made some success to progress the TOE. In terms of the theory for handling uncertainty, the author recommends the quantum decision theory (QDT) developed by Yukalov \& Sornette $[16,17]$ which can consider the preferences of the individuals/players. The decision makers' brain is assumed of a dual nature, with brain processes divided to conscious and subconscious constituent parts and the computed quantum strategic probabilities consisted of two parts, the first one being rationality related and the other one capturing biases, emotions and feelings.

\section{Summary and Conclusion}

In this paper, a novel cosmological model based on Buddhist philosophy is proposed and from this author's judgement, it seems conceptually clear and logically consistent. It can overcome many deficiencies of the current Big-Bang cosmological model and explain many phenomena which belongs to the frontier problems of modern sciences. One important feature is that if this BCM is proved to be valid, it may have great impact on human behavior since nowadays most of the people believe in the materialism which is the cause of many human conflicts for the limited resources.

\section{Acknowledgement}

This work was supported by the State Key Program of National Natural Science of China "Structural Reliability Analysis on the Spherical Hull of Deepsea Manned Submersibles" (Grant No. 51439004), the General Program of National Natural Science of China "A study on the water absorption property of the buoyancy material for the full ocean depth manned submersible" (Grant No. 51879157), the "Construction of a Leading Innovation Team" project by the Hangzhou Municipal government, the Startup funding of New-joined PI of Westlake University with grant number (041030150118).

\section{References}

1. Uzan, Jean-Philippe (2015) The Big-Bang Theory: Construction, Evolution and Status L'Univers, S'eminaire Poincar'e XX: 1-69.

2. Burago, SG (2017) About the theory of the Big Bang, The General Science Journal. Astrophysics. p. 1-7.

3. Moreira-Almeida, A, Santos, FS (2012) Exploring Frontiers of the Mind-Brain Relationship, Springer, New York, Dordrecht Heidelberg, London.

4. Sheldrake, R (2012) The Science Delusion Freeing the Spirit of Enquiry. Coronet, An imprint of Hodder \& Stoughton, A Hachette UK company.

5. Tart, CT (2009) The End of Materialism: How Evidence of the Paranormal Is Bringing Science and Spirit Together, New Harbinger Publications, Inc, Oakland, USA.

6. Kant, I (2002) Metaphysical Foundations of Natural Science, in Theoretical Philosophy after 1781, edited by Henry Allison and Peter Heath, translated by Michael Friedman, Cambridge: Cambridge University Press.

7. Laumakis, Stephen, J (2008) An Introduction to Buddhist Philosophy. Cambridge University Press, New York, USA.

8. Harvey, P (2013) An Introduction to Buddhism, Teachings, History and Practices, 2nd Edition, Cambridge University Press, Cambridge, New York, Melbourne, Madrid, Cape Town, Singapore, São Paulo, Delhi, Mexico City.

9. Das, S (2013) Reincarnation-a Law of Nature, International Journal of Modern Engineering Research (IJMER) 3(2): 1192-1211.

10. Ingram, DM (2007) Mastering the teachings of the Budda, An Unusually Hardcore Dharma Book, Third Edition, Published by the Interdependent Universe.

11. Silver, D (2017) Mastering the game of GO without human knowledge. Nature 550: 354-371.

12. Whitaker, A (2006) Einstein, Bohr and the Quantum Dilemma - From Quantum Theory to Quantum Information ( $2^{\text {nd }}$ Edn), Paperback, Cambridge University Press, UK.

13. Cui, W.C., Kang, BL (2008) Examining the debate between Einstein and Bohr based on Buddhist philosophy. Journal of Chinese Social Sciences 55(8): 1-9 (in Chinese). 


\section{Annals of Social Sciences \& Management studies}

14. Cui, W.C. (2019) A Simple Idea on the Unification of Einstein-Bohr Controversy. Ann Soc Sci Manage Stud. 2(5): 555597.

15. Shen, Zhi-Yuan (2013) Stochastic Quantum Space Theory on Particle Physics and Cosmology-A New Version of Unified Field Theory, Journal of Modern Physics 4: 1213 -1380.
16. Yukalov, VI, Sornette, D (2011) Decision Theory with Prospect Interference and Entanglement. Theory and Decision 70: 283-328.

17. Yukalov, VI, Sornette, D (2016) Quantum Probability and Quantum Decision-making. Phil. Trans. R. Soc A 374: 1-15.

Your next submission with Juniper Publishers
will reach you the below assets
- Quality Editorial service
- Swift Peer Review
- Reprints availability
- E-prints Service
- Manuscript Podcast for convenient understanding
- Global attainment for your research
- Manuscript accessibility in different formats
( Pdf, E-pub, Full Text, Audio)
- Unceasing customer service
Track the below URL for one-step submission
https://juniperpublishers.com/online-submission.php

